\title{
On Linear Approximation of Modulo Sum
}

\author{
Alexander Maximov \\ Department of Information Technology \\ Lund University \\ Box 118, SE-22100 Lund, Sweden \\ movax@it.lth.se
}

\begin{abstract}
The general case for a linear approximation of the form " $X_{1}+\cdots+X_{k} \bmod 2^{n "} \rightarrow$ " $X_{1} \oplus \cdots \oplus X_{k} \oplus N$ " is investigated, where the variables and operations are $n$-bit based, and the noise variable $N$ is introduced due to the approximation. An efficient and practical algorithm of complexity $O\left(n \cdot 2^{3(k-1)}\right)$ to calculate the probability $\operatorname{Pr}\{N\}$ is given, and in some cases it can be reduced to $O\left(2^{k-2}\right)$.
\end{abstract}

\section{Introduction}

Linear approximations of nonlinear blocks in a cipher is a common tool for cryptanalysis. One of the most typical approximations is the substitution of the arithmetical sum modulo $2^{n}(\boxplus)$ with the XOR-operation $(\oplus)$ of the input variables. We introduce a noise variable $N$ and write: $X_{1} \boxplus \cdots \boxplus X_{k}=X_{1} \oplus \cdots \oplus$ $X_{k} \oplus N$. For a distinguishing attack the bias of a linear combination of noise variables can be calculated if their distributions are known. For the considered approximation the distribution of $N$ can be calculated in two ways:

I. for $X_{1}=0 \ldots 2^{n}-1 \quad \leftarrow O\left(2^{k \cdot n}\right)$

$$
\begin{aligned}
& \ddots \\
& \text { for } X_{k}=0 \ldots 2^{n}-1 \\
& \operatorname{Dist}_{N}\left[\left(X_{1} \boxplus \cdots \boxplus X_{k}\right) \oplus\right. \\
& \left.\quad\left(X_{1} \oplus \cdots \oplus X_{k}\right)\right]++;
\end{aligned}
$$

or

II. for $C=0 \ldots 2^{n}-1 \quad \leftarrow O\left(c \cdot 2^{n}\right)$

$\operatorname{Dist}_{N}[\mathrm{C}]=\operatorname{ProbOfN}(C)$;

where the function ProbOfN $(C)$ calculates the corresponding probability (see Section 2). Note that we deal with integer-valued distribution tables, i.e., $\operatorname{Pr}\{N=C\}=\operatorname{Dist}_{N}[C] / 2^{k \cdot n}$.

\section{The Function ProbOfN $(C)$}

Let $C=\overline{c_{n} \ldots c_{2} 0}$ (note that $\operatorname{Pr}\left\{N=\overline{c_{n} \ldots c_{2} 1}\right\}=0$ ). Then:

$$
\operatorname{ProbOfN}(C)=\left(\begin{array}{lll}
1 & 1 \ldots 1
\end{array}\right) \times \prod_{i=n}^{2} \mathbf{T}_{c_{i}} \times \mathbf{S}_{\mathbf{0}},
$$


where $\mathbf{T}_{\mathbf{0}}, \mathbf{T}_{\mathbf{1}}$, and $\mathbf{S}_{\mathbf{0}}$ are fixed matrices. The algorithm to construct the matrices $\mathbf{T}_{\mathbf{0}}, \mathbf{T}_{\mathbf{1}}$, and $\mathbf{S}_{\mathbf{0}}$ is given below.

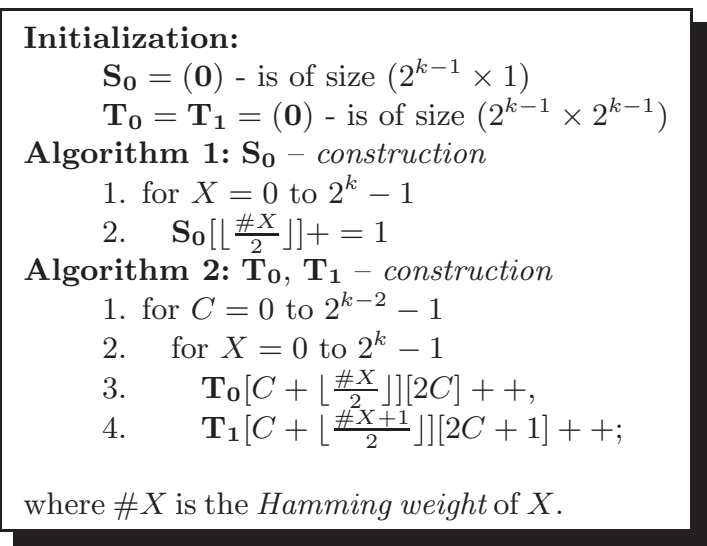

\section{Example}

Assume that $n=5$ and $k=3$, i.e., $N=\left(X_{1} \boxplus X_{2} \boxplus X_{3}\right) \oplus\left(X_{1} \oplus X_{2} \oplus X_{3}\right)$. Then:

$$
\mathbf{T}_{\mathbf{0}}=\left(\begin{array}{llll}
4 & 0 & 0 & 0 \\
4 & 0 & 4 & 0 \\
0 & 0 & 4 & 0 \\
0 & 0 & 0 & 0
\end{array}\right) \mathbf{T}_{\mathbf{1}}=\left(\begin{array}{llll}
0 & 1 & 0 & 0 \\
0 & 6 & 0 & 1 \\
0 & 1 & 0 & 6 \\
0 & 0 & 0 & 1
\end{array}\right) \mathbf{S}_{\mathbf{0}}=\left(\begin{array}{l}
4 \\
4 \\
0 \\
0
\end{array}\right) \text {. }
$$

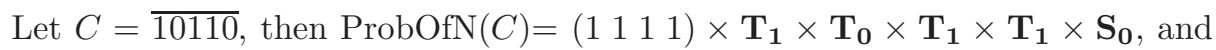
$\Rightarrow \operatorname{Pr}\{N=\overline{10110}\}=1536 / 2^{3 \cdot 5}=0.046875$.

\section{Optimization Ideas}

If $n$ is not very large, say $n=32$ bits, then optimization can be done in the following way. Represent $C=\overline{A B 0}$, where $A=\overline{c_{32} \ldots c_{16}}$ and $B=\overline{c_{15} \ldots c_{2}}$. Then create two tables of vectors: $R_{\text {Left }}[A]=\left(\begin{array}{lll}1 & 1 \ldots 1\end{array}\right) \times \prod_{i=32}^{16} \mathbf{T}_{c_{i}}$ and $R_{\text {Right }}[B]=$ $\prod_{i=15}^{2} \mathbf{T}_{c_{i}} \times \mathbf{S}_{\mathbf{0}}$, for all $A$ and $B$. Then the probability $\operatorname{Pr}\{N=C\}$ is just a scalar product $R_{\text {Left }}\left[\overline{c_{32} \cdots c_{16}}\right] \times R_{\text {Right }}\left[\overline{c_{15} \cdots c_{2}}\right]$, and the time complexity is $O\left(2^{k-2}\right)$. This idea of partitioning can be extended to larger $n$ as well. 